Original

\title{
Ghrelin directly stimulates adult hippocampal neurogenesis: implications for learning and memory
}

\author{
Endan $\mathrm{Li}^{1)}$, Hyunju Chung ${ }^{2)}$, Yumi Kim ${ }^{1)}$, Dong Hyun $\mathrm{Kim}^{3)}$, Jong Hoon Ryu ${ }^{3)}$, Takahiro Sato ${ }^{4)}$, \\ Masayasu Kojima ${ }^{4)}$ and Seungjoon Park ${ }^{1)}$ \\ 1) Department of Pharmacology and Medical Research Center for Bioreaction to ROS and Biomedical Science Institute, School of \\ Medicine, Kyung Hee University, Seoul,Koera \\ 2) Department of Core Research Laboratory, Clinical Research Institute, Kyung Hee University Hospital at Gangdong, Seoul, \\ Korea \\ 3) Department of Oriental Pharmaceutical Science and Kyung Hee East-West Pharmaceutical Research Institute, College of \\ Pharmacy, Kyung Hee University, Seoul, Korea \\ 4) Department of Molecular Genetics, Institute of Life Science, Kurume University, Fukuoka, Japan
}

\begin{abstract}
Adult hippocampal neurogenesis is important in mediating hippocampal-dependent learning and memory. Exogenous ghrelin is known to stimulate progenitor cell proliferation in the dentate gyrus of adult hippocampus. The aim of this study was to investigate the role of endogenous ghrelin in regulating the in vivo proliferation and differentiation of the newly generating cells in the adult hippocampus using ghrelin knockout (GKO) mice. Targeted deletion of ghrelin gene resulted in reduced numbers of progenitor cells in the subgranular zone (SGZ) of the hippocampus, while ghrelin treatment restored progenitor cell numbers to those of wild-type controls. We also found that not only the number of bromodeoxyuridine (BrdU)-positive cells but also the fraction of immature neurons and newly generated neurons were decreased in the GKO mice, which were increased by ghrelin replacement. Additionally, in the GKO mice, we observed impairment of memory performance in Y-maze task and novel object recognition test. However, these functional deficiencies were attenuated by ghrelin administration. These results suggest that ghrelin directly induces proliferation and differentiation of adult neural progenitor cells in the SGZ. Our data suggest ghrelin may be a plausible therapeutic potential to enhance learning and memory processes.
\end{abstract}

Key words: Ghrelin, Hippocampus, Neurogenesis, Learning, Memory

MAMMALIAN NEUROGENESIS persists into adulthood in several species, including humans, in the subventricular zone of the lateral ventricle and in the subgranular zone (SGZ) of the dentate gyrus (DG) in the hippocampus $[1,2]$. There has been much attention on neurogenesis in the hippocampus in the normal brain because this structure is important in the process of learning, memory and emotional responses [2]. Several endogenous growth factors, such as fibroblast growth factor-2 (FGF-2), insulin-like growth factor-1 (IGF-1) and vascular endothelial growth factor (VEGF), improve cognitive function either by direct effects on the generation of new neurons or indirectly through neurotrophic

Submitted Jan. 5, 2013; Accepted Jan. 29, 2013 as EJ13-0008 Released online in J-STAGE as advance publication Feb. 15, 2013

Correspondence to: Seungjoon Park, M.D., Ph.D., Department of Pharmacology and Medical Research Center for Bioreaction to ROS and Biomedical Science Institute, School of Medicine, Kyung Hee University, Seoul 130-701, Korea. E-mail: sjpark@khu.ac.kr effects that promote the survival of new neurons in the hippocampus [3]. However, the endogenous factors that regulate the proliferation of neural progenitor cells in the adult hippocampus need to be further clarified.

Several studies have demonstrated that ghrelin enhances neurogenesis. Initially, ghrelin was shown to increase neurogenesis in the dorsal motor nucleus of vagus [4], the nucleus of the solitary tract [5] and the rat fetal spinal cord [6] in adult rats. We recently have reported that systemic administration of ghrelin stimulates proliferation of newly generating cells in the hippocampus of adult mice [7]. Moreover, immunoneutralization of ghrelin by using anti-ghrelin antiserum in adult mice reduces proliferation of hippocampal progenitor cells in the SGZ [7]. These findings prompted us to hypothesize that endogenous ghrelin may play an important role in adult hippocampal neurogenesis. Considering that neurogenesis in the DG has been proposed to mediate hippocampal-dependent learning and 
memory [2], ghrelin-induced stimulation of neurogenesis in hippocampal progenitor cells may play an important role in the ability of ghrelin to enhance memory performance. Indeed, ghrelin knockout (GKO) mice showed impaired memory performance, which was reversed by peripheral administration of ghrelin [8]. However, it is still unclear whether ghrelin directly regulates adult neurogenesis in the DG of the hippocampus. In addition, it remains to be determined that ghrelin-induced neurogenesis is functionally important in learning and memory.

Therefore, in this study, we investigated the role of endogenous ghrelin in regulating the in vivo proliferation and differentiation of the newly generating cells in the adult hippocampus by using ghrelin-deficient mice. We also examined the effect of ghrelin on behavior of these animals in the Y-maze task and the novel object recognition (NOR) test.

\section{Materials and Methods}

\section{Animals}

To determine the role of endogenous role of ghrelin in adult hippocampal neurogenesis, we used 8-9 weekold male GKO mice [9] and age-matched wild-type C57BL/6J mice. The animals were housed under controlled environmental conditions (12-h light and 12-h dark) with free access to food and water. They were habituated to the housing conditions for 7-10 days prior to the beginning of the experimental procedures. All experiments were approved by the Kyung Hee University Animal Care Committee and conducted according to the principles and procedures outlined in the Guide for the Care and Use of Laboratory Animals as adopted and promulgated by the U.S. National Institutes of health. All efforts were made to minimize animal suffering and to reduce the number of animals used.

\section{Ghrelin treatment and bromodeoxyuridine (BrdU) injection}

Ghrelin (Peptides International, Inc., Louisville, $\mathrm{KY}$ ) at a dose of $80 \mu \mathrm{g} / \mathrm{kg}$ was used to study its effect on progenitor cell proliferation or differentiation in the adult DG of the hippocampus. This dose of ghrelin was chosen on the basis of our previous study demonstrating its effectiveness on cell proliferation in the DG of adult mice [7]. GKO mice received ghrelin or saline intraperitoneally once daily for 8 consecutive days. The wild-type mice were given saline at corresponding times. BrdU (50 mg/kg; Sigma) was given twice daily at 8 -h intervals during the last 3 days of ghrelin treatment, and mice were transcardially perfused 1,7 or 28 day(s) after the last BrdU injection (for the evaluation of BrdU-labeled cells).

\section{Tissue preparation and immunohistochemistry}

Animals were anesthetized with xylazine and ketamine and then perfused transcardially with a freshly prepared solution of $4 \%$ paraformaldehyde in phosphate-buffered saline (PBS). The brains were removed and post-fixed overnight in the same fixative before being immersed in a solution of $30 \%$ sucrose in PBS. Serial $30-\mu \mathrm{m}$-thick coronal tissue sections were cut using a microtome and stored in cryoprotectant $(25 \%$ ethylene glycol, $25 \%$ glycerol, $0.05 \mathrm{M} \mathrm{PB}$; $\mathrm{pH} 7.4$ ) at $-20{ }^{\circ} \mathrm{C}$ for later immunohistochemistry procedures.

In order to minimize staining variations, the brain sections from the same experiment were processed for immunohistochemical staining simultaneously. To ensure the detection of BrdU-labeled nuclei, we denatured the DNA before incubation with BrdU antibody because BrdU is incorporated into the DNA. DNA denaturation was performed in the following manner: tissue was incubated in $50 \%$ formamide and $2 \times \mathrm{SSC}(1 \times \mathrm{SSC}$, $0.3 \mathrm{M} \mathrm{NaCl}$ and $0.03 \mathrm{M}$ sodium citrate) for $2 \mathrm{~h}$ at $65^{\circ} \mathrm{C}$, rinsed for $15 \mathrm{~min}$ in $2 \times \mathrm{SSC}$, incubated again for $10 \mathrm{~min}$ in $0.1 \mathrm{M}$ boric acid at $\mathrm{pH}$ 8.5. Free-floating tissue sections were then incubated overnight at $4{ }^{\circ} \mathrm{C}$ with primary antibodies (rat anti-BrdU antibody, 1:1000, Santa Cruz Biotechnology, Inc., Santa Cruz, CA; mouse anti-proliferating cell nuclear antigen (PCNA) antibody, 1:500, DakoCytomation, Denmark; rabbit anti-Ki-67 antibody, 1:1000, Abcam, Cambridge, UK). The sections were incubated with appropriate biotinylated secondary antibody (1:200, Vector Laboratories, Burlingame, CA) and then visualized using the avidin-biotin-peroxidase complex method with diaminobenzidine tetrahydrochloride (DAB) as the chromogen. For immunodetection of BrdU, the DAB-nickel enhancement technique was used. For detection of Ki-67, the sections were incubated with a secondary $\mathrm{Cy} 3$-goat anti-rabbit IgG (1:400, Jackson ImmunoResearch, West Grove, PA).

To determine the phenotypes of BrdU-labeled cells, brain sections were processed for BrdU, doublecortin (DCX), neuron-specific nuclear antigen $(\mathrm{NeuN})$, or glial fibrillary acidic protein (GFAP) fluorescent double labeling. Briefly, free-floating brain sections were first pretreated for DNA denaturation as described above, and 
then incubated overnight at $4{ }^{\circ} \mathrm{C}$ with rat anti-BrdU antibody (1:1000) and goat anti-DCX (1:1000, Santa Cruz Biotechnology, Inc.), mouse anti-NeuN (1:1000, Millipore, Billerica, MA) or rabbit anti-GFAP (1:1000, DakoCytomation). After washing in 0.1 M PBS, sections were incubated for $1.5 \mathrm{~h}$ with fluorescent secondary antibodies: Alexa Fluor 488 goat anti-rat $\operatorname{IgG}$ to reveal immunoreactivity of $\mathrm{BrdU}$ and $\mathrm{Cy} 3$-conjugated affinipure donkey anti-goat IgG, Cy3-conjugated affinipure goat anti-mouse IgG or Cy3-conjugated affinipure goat anti-rabbit $\operatorname{IgG}$ (1:400, Jackson ImmunoResearch) to reveal immunoreactivities of DCX, NeuN or GFAP, respectively. Then, the sections were washed in PBS and mounted onto coated glass slides, and coversliped with fluorescent mounting medium.

\section{Quantification and phenotype of newborn cells}

The total numbers of BrdU-, PCNA- and Ki-67labeled cells in mice were manually counted in the right and left SGZ along the superior and inferior blades of the DG of every sixth sections throughout the entire extent of the hippocampus (from Bregma -1.06 $\mathrm{mm}$ to Bregma $-3.80 \mathrm{~mm}$ ), using Stereo Investigator (MicroBrightField, Williston, VT). Analysis was performed using a computer-assisted image analysis system consisting of a Zeiss Axioscope-2 microscope equipped with a computer-controlled motorized stage, a video camera, and Stereo Investigator software. Counting was done at $400 \times$ magnification using a Zeiss Axioscope- 2 microscope equipped with a $40 \times$ objective lens. Cells were counted throughout each section by focusing through each focal plane of the section to ensure that all BrdU-labeled cells were visible. For PCNA and Ki-67, only cells with robust nuclear immunoreactivity were considered positive. The resulting numbers from each section were added and multiplied by 6 to obtain the estimated total number of labeled cells per DG for each brain.

Double-labeled cells for BrdU and DCX, NeuN or GFAP were counted using a Carl Zeiss LSM 700 Meta confocal microscope. The extent of colocalization was confirmed by three-dimensional reconstructions (X, Y, and $Z$ ) using $Z$-plane sectioning in order to exclude false double labeling. BrdU single-labeled cells and BrdU/ DCX, BrdU/NeuN or BrdU/GFAP double-labeled cells were counted. The percentage of BrdU cells doublelabeled for DCX, NeuN or GFAP was calculated by dividing the number of double-labeled cells by the total number of BrdU cells and multiplying 100.

\section{Behavioral analysis}

The Y-maze is a three-arm maze with equal angles between all arms. The maze floor and walls were constructed from dark opaque polyvinyl plastic. Animals were initially placed within one arm, and the sequence and number of arm entries were recorded manually for each animal over an $8 \mathrm{~min}$ period. The percentage of trials in which all three arms were represented, i.e., $\mathrm{ABC}$, $\mathrm{CAB}$, or $\mathrm{BCA}$ but not $\mathrm{BAB}$, was recorded as an alternation to estimate of short-term memory. Arms were cleaned between tests to remove odors and residues. The alternation score (\%) for each animal was defined as the ratio of the actual number of alternation to the possible number (defined as the total number of arm entries minus two) multiplied by 100 as shown by the following equation: \% Alternation $=[($ Number of alternations $) /($ Total arm entries -2$)] \times 100$. The number of arm entries was used as an indicator of locomotor activity.

We also measured performance of wild-type and GKO mice on NOR, a type of memory considered to be dependent upon the hippocampus [10,11]. During the training phase, animals were first allowed to explore two identical objects (A and B) in a test chamber for 5 $\min$. The exploration time for each object was recorded using a video-based Ethovision System (Nodulus, Wageningen, The Netherlands). After a 24-h delay, animals were placed again in the apparatus, where one of the original objects was replaced by a novel one (C), and exploration time was measured again for $5 \mathrm{~min}$.

For the Y-maze task and NOR test, animals were implanted with subcutaneous Alzet osmotic minipumps (DURECT Corporation, Cupertino, CA) delivering either ghrelin $(50 \mu \mathrm{g} / \mathrm{kg}$ per day ghrelin dissolved in saline, $n=6)$ or vehicle $(n=6)$ for 28 days. Wild-type mice $(n=6)$ were given saline via minipumps.

\section{Statistical analysis}

Data are presented as mean \pm SEM. Statistical analysis between groups was performed using 1-way ANOVA and Holm-Sidak method for multiple comparisons using SigmaStat for Windows Version 3.10 (Systat Software, Inc. Point Richmond, CA). P $<0.05$ was considered statistically significant.

\section{Results}

\section{Effect of ghrelin on hippocampal neurogenesis in adult mice}

Our previous report [7] demonstrating the prolifera- 
tive effect of ghrelin and the inhibitory effect of antighrelin antibody on hippocampal progenitor cells in normal adult mice suggests the role of endogenous ghrelin in neurogenesis. To address whether endogenous ghrelin controls proliferation of neural precursor cells, we assessed the number of cycling cells in the DG region of GKO mice and their wild-type controls by using PCNA, a marker for cell proliferation [1]. We detected significantly lower number of newly generating PCNA-labeled cells in GKO mice compared to wild-type controls (Fig. 1A, B), indicating decreased cell proliferation. To determine whether exogenous ghrelin can alter the number of PCNA-labeled cells in the DG of GKO mice, we assessed this parameter after treatment of GKO mice with ghrelin $(80 \mu \mathrm{g} / \mathrm{kg}$, ip, once daily for 8 consecutive days). This treatment resulted in a shift in the number of newborn cells of GKO animals toward the wild-type values (Fig. 1A, B). We also examined the changes in the number of Ki-67-labeled cells, another marker for cell proliferation in the brain [12], and obtained similar results (Fig. 1A, C).

Consistent with these findings, we found similar changes in the number of BrdU-labeled cells in the DG 1 day after the last BrdU injection (Fig. 1A, D). In order to ascertain the phenotype of newborn (proliferating) cells labeled by BrdU under these conditions,

A

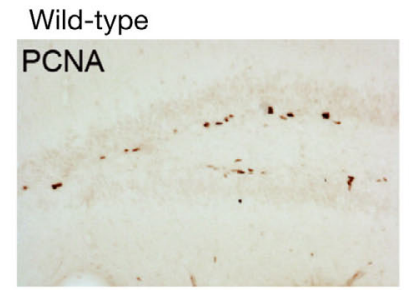

GKO+Vehicle

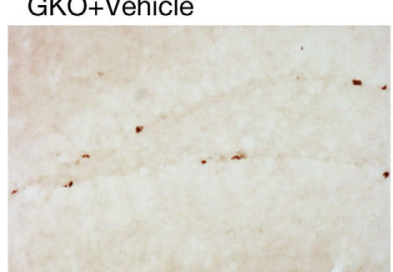

GKO+Ghrelin
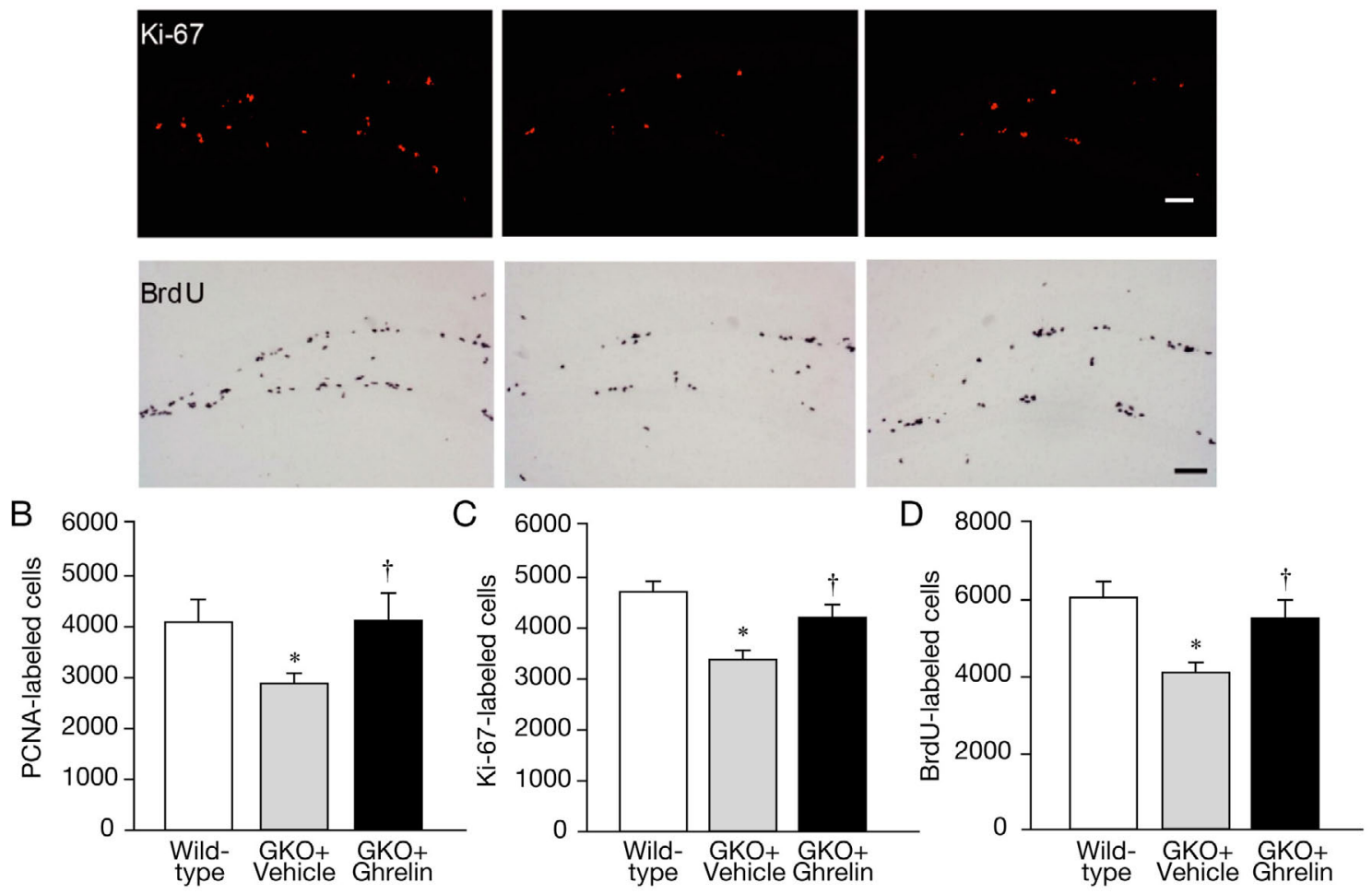

Fig. 1 Effect of ghrelin on cell proliferation in the DG of adult mice. Animals were injected intraperitoneally with vehicle or ghrelin $(80$ $\mu \mathrm{g} / \mathrm{kg}$ ) once daily for 8 consecutive days. BrdU (50 mg/kg, ip) was administered twice daily during the last 3 days of ghrelin treatment and transcardially perfused 1 day after the last BrdU injection. The number of PCNA-, Ki-67- or BrdU-labeled cells was counted in the subgranular zone of the DG. A, PCNA- (upper panel), Ki-67- (middle panel) and BrdU-labeled (lower panel) cells in the hippocampus. Scale bar represents $100 \mu \mathrm{m}$ for PCNA and BrdU or $50 \mu \mathrm{m}$ for Ki-67. B-D, Quantitative analysis of PCNA- (B), Ki-67- (C) and BrdU-labeled (D) cells in the hippocampus. The data expressed as the mean \pm SEM ( $\mathrm{n}=5 /$ group). $* P<0.05 v s$. wild-type, $\dagger P<0.05 v s$. vehicle-treated GKO mice. 
A

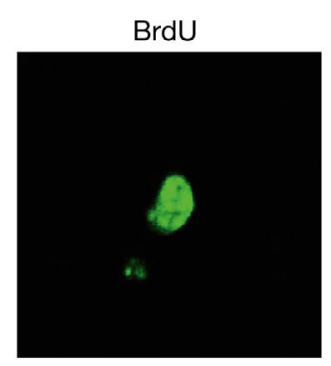

B

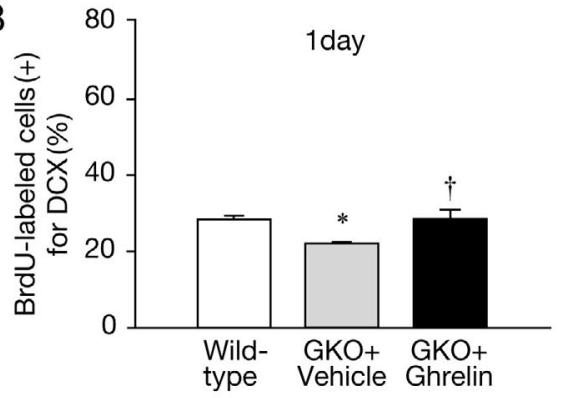

DCX
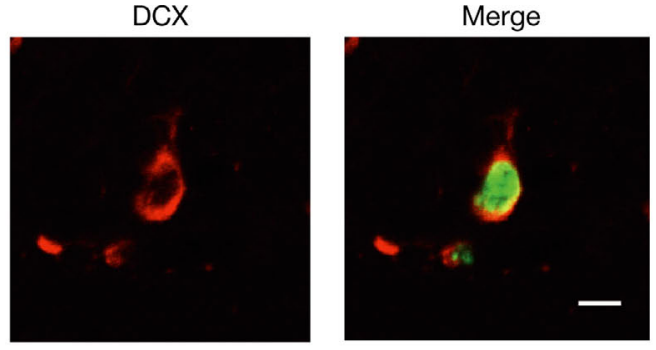

C

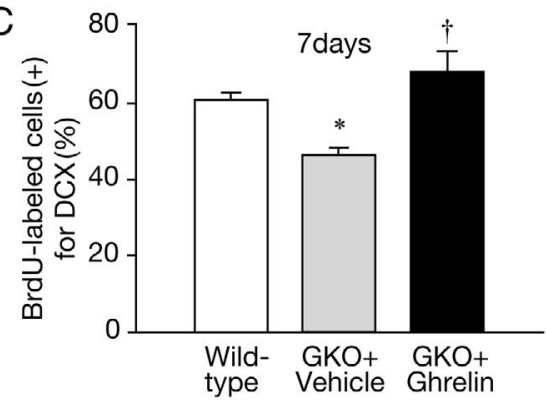

Fig. 2 Effect of ghrelin on the percentage of BrdU/DCX double-labeled cells in the DG of adult mice. Animals were injected intraperitoneally with vehicle or ghrelin $(80 \mu \mathrm{g} / \mathrm{kg})$ once daily for 8 consecutive days. BrdU (50 mg/kg, ip) was administered twice daily during the last 3 days of ghrelin treatment and transcardially perfused 1 day (B) or 7 days (C) after the last BrdU injection. A, Confocal microscopic images showing co-localization of BrdU with DCX. Scale bar represents $5 \mu \mathrm{m}$. B and C, Quantitative analysis of the percentage of BrdU-labeled cells double-labeled for DCX 1 day (B) or 7 days (C) after BrdU labeling. The data expressed as the mean $\pm \mathrm{SEM}$ (n=5/group). ${ }^{*} P<0.05$ vs. wild-type, $\dagger P<0.05$ vs. vehicle-treated GKO mice.

brain sections were stained with antibodies against both BrdU and the early neuronal marker DCX. Double labeling immunohistochemistry showed co-expression of nuclear BrdU and cytoplasmic DCX in the DG (Fig. $2 \mathrm{~A})$. One day after the last BrdU injection, the percentage of BrdU/DCX double-labeled cells was significantly reduced in GKO mice (21.7\%) compared to wild-type controls (28.4\%; Fig. 2B). However, ghrelin treatment restored the percentage of the BrdU/DCX double-labeled cells of GKO mice to the control values (Fig. 2B). We also found a similar reduction in the percentage of BrdU-labeled cells co-expressing DCX in GKO mice $(46.0 \%)$ than in wild-type controls $(60.1 \%)$ 7 days after the last BrdU administration, which was significantly increased by ghrelin treatment (Fig. 2C).

Next, we determined if ghrelin could affect the fate of newborn cells. The total number of BrdU-labeled cells remained significantly lower in the GKO mice than the wild-type controls 28 days after the last BrdU administration (Fig. 3C). By contrast, ghrelin treatment resulted in an increase in the number of BrdU-labeled cells toward the wild-type values (Fig. 3C). The extent of differentiation of survived BrdU-labeled cells was determined by double labeling immunohistochemis- try with antibodies against $\mathrm{BrdU}$ and $\mathrm{NeuN}$, the neuronal marker, or GFAP, the glial marker (Fig. 3A, B). Confocal microscopic analysis of colocalization of BrdU with NeuN or GFAP revealed that the majority of BrdU-labeled cells had the neuronal phenotype and a low percentage of BrdU-labeled cells were positive for GFAP (Fig. 3D). The percentage of BrdU-labeled cells that were double labeled for NeuN was significantly decreased in GKO mice, which was increased to wild-type values when GKO animals were treated with ghrelin (Fig. 3D). In contrast, the percentage of BrdUand GFAP-labeled cells was not altered either in GKO mice or by ghrelin treatment (Fig. 3D).

\section{Effect of ghrelin on learning and memory processes in adult mice}

To investigate whether endogenous ghrelin plays a physiologic role in hippocampus-dependent spatial working memory performance, animals were tested for spontaneous alternation behavior in a Y-maze. There was no difference in total entry between groups (Fig. 4A). GKO mice showed significantly reduced alternation rates when compared to wild-type controls (Fig. 4B). In contrast, ghrelin replacement by subcutaneous 
A
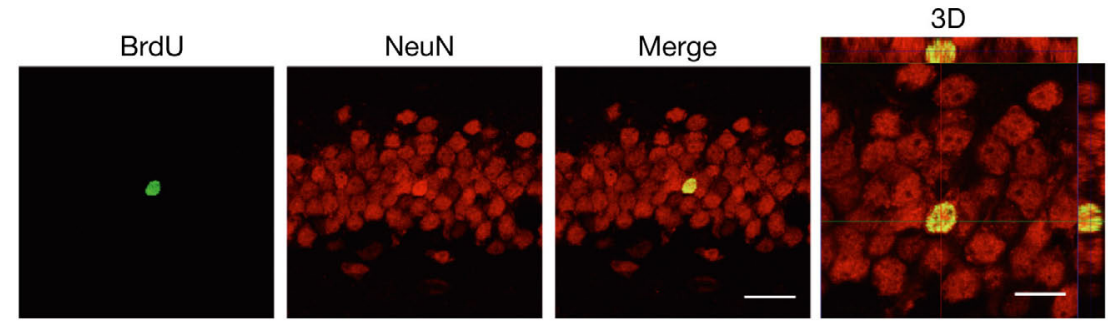

B

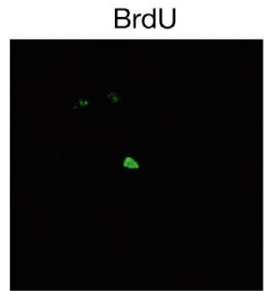

GFAP
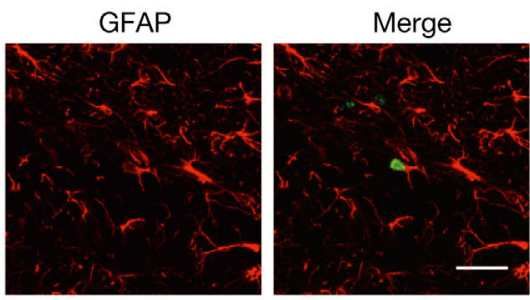

3D

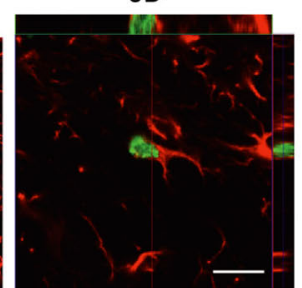

C

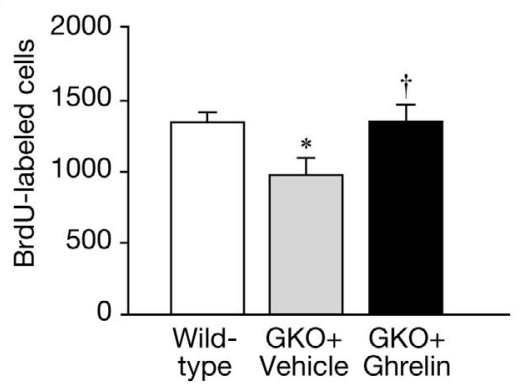

D

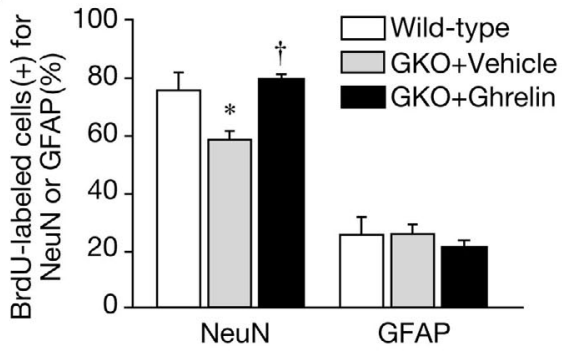

Fig. 3 Effect of ghrelin on cell differentiation in the DG of adult mice. Animals were injected intraperitoneally with vehicle or ghrelin $(80 \mu \mathrm{g} / \mathrm{kg})$ once daily for 8 consecutive days. BrdU (50 mg/kg, ip) was administered twice daily during the last 3 days of ghrelin treatment and transcardially perfused 28 days after the last BrdU injection. A and B, Confocal microscopic images showing colocalization of BrdU with NeuN (A) or GFAP (B). Scale bar represents $10 \mu \mathrm{m}$. C, Quantitative analysis of BrdU-labeled cells 28 days after BrdU labeling. D, Quantitative analysis of the percentage of BrdU-labeled cells double labeled for NeuN or GFAP.

The data expressed as the mean \pm SEM ( $\mathrm{n}=5$ /group). $* P<0.05 v$ s. wild-type, $\uparrow P<0.05 v s$. vehicle-treated GKO mice.
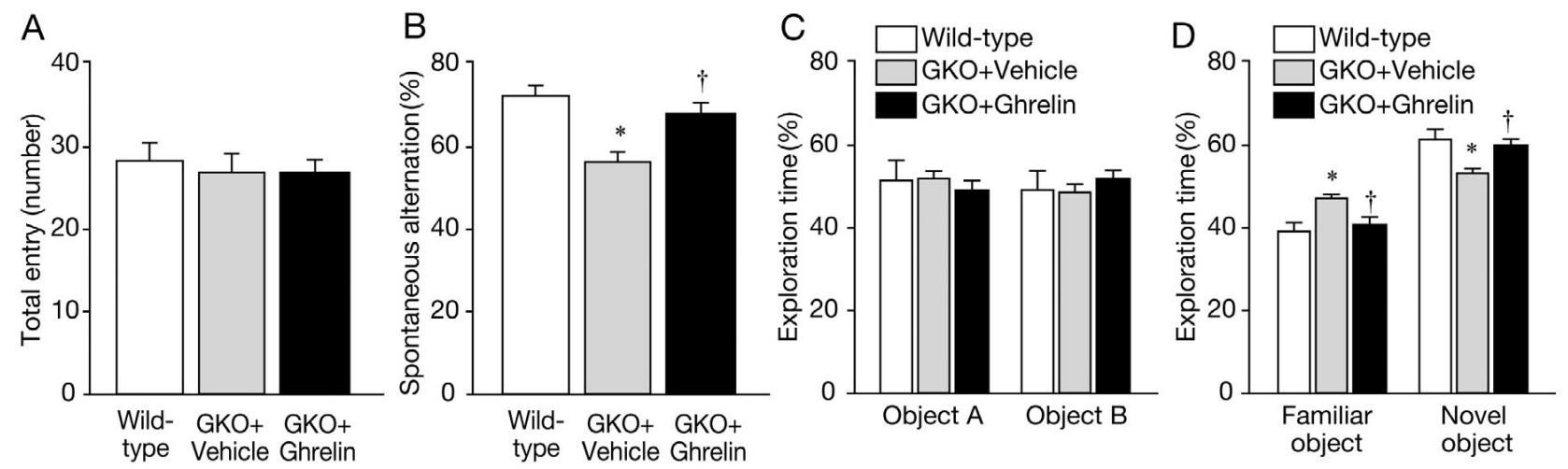

Fig. 4 Effect of ghrelin on memory performance of GKO mice evaluated by the Y-maze task (A and B) and the novel objective recognition test $(\mathrm{C}$ and $\mathrm{D})$. GKO mice were treated with ghrelin $(50 \mu \mathrm{g} / \mathrm{kg} / \mathrm{day})$ or vehicle (saline) by subcutaneous osmotic minipumps for 28 days. Wild-type mice were given saline via minipumps. A, Total arm entries in the Y-maze. B, Spontaneous alternation behavior in the Y-maze. The GKO mice showed a significant decrease in alternation behavior, which was reversed by ghrelin administration. C, Exploration time during the first phase. All three groups spent similar amounts of time at each objects (A and B). D, Exploration time during the second phase. GKO mice spent more time exploring the familiar object and less time exploring the novel object when compared to wild-type mice. These differences were abolished by 28 days of ghrelin replacement. The data expressed as the mean \pm SEM (n=6/group). ${ }^{*} P<0.05$ vs. wild-type, $\uparrow P<0.05$ vs. vehicle-treated GKO mice. 
osmotic mini-pump for 4 weeks significantly attenuated this impairment (Fig. 4B).

We also measured how GKO mice performed in the NOR test that is used to assess non-spatial memory and relies on the animal's natural tendency to differentially explore novel objects over familiar ones. The NOR is known to depend, in part, on hippocampus $[10,11]$. In the first phase of the test, there was no significant difference in total exploration time between groups and all three groups spent similar amounts of time at each object (A or B) (Fig. 4C). After a 24-h delay, the animals were exposed to two dissimilar objects placed in the same test chamber: one familiar object (A or B) used in the first phase and one novel object $(\mathrm{C})$, and the time spent exploring each of the objects was measured. As shown in Fig. 4D, wild-type animals spent more time exploring the novel object than the familiar one. In contrast, GKO mice showed significantly increased exploration time for familiar object and decreased exploration time for novel one when compared to wildtype animals. These data suggest that GKO mice have impairment in their memory of the previous objects. However, this functional deficiency was disappeared when GKO mice were replaced with ghrelin by subcutaneous osmotic mini-pumps (Fig. 4D).

\section{Discussion}

Over the last decade, numerous studies have demonstrated that ghrelin plays important roles in GH release, food intake, body weight regulation and glucose homeostasis. Besides its importance in energy metabolism, the abundant expression of GHS-R1a in brain regions outside the hypothalamus suggests its significance in neuronal function. Emerging evidence suggests that ghrelin acts in the CNS to control neuronal function and subsequently has a profound influence on various brain functions [13]. In the current study, we provide evidence that endogenous ghrelin regulates adult hippocampal neurogenesis and it is important for ghrelin-induced learning and memory processes. Specifically, ghrelin-deficient mice showed lower numbers of progenitor cells in the DG of the hippocampus, while ghrelin treatment restored progenitor cell numbers to those of wild-type controls. To the best of our knowledge, this is the first report demonstrating reduced adult hippocampal neurogenesis in ghrelin-deficient mice.

Given that peripheral ghrelin can pass through the blood-brain barrier and ghrelin binding sites and receptors are found in the hippocampus [8] strongly suggests that peripherally administered ghrelin enters the hippocampus and directly stimulates neurogenesis within the DG of the hippocampus. Our results also showed that differentiation is affected by ghrelin, because a decrease was seen not only in the number of BrdU-positive cells but also in the fraction of immature neurons and newly generated neurons in the GKO mice, which were increased by ghrelin replacement. Nonetheless, we cannot exclude the possibility that the action of ghrelin could be, in part, due to the ability of ghrelin to stimulate the GH/IGF-1 axis [14], where both GH and IGF-1 infusions have been shown to increase hippocampal neurogenesis $[15,16]$.

The ghrelin-associated changes in hippocampal neurogenesis observed in experimental models may indeed be important in learning and memory process. For example, calorie restriction, which is paralleled by elevated circulating ghrelin [17], is known to be associated with increased adult neurogenesis in young adult rats [18] and enhanced performance of animals in a variety of behavioral tests that are dependent on the hippocampus $[19,20]$. In contrast, the development of dementia is more common in the elderly and obese patient [21], which are known to have reduced ghrelin levels $[22,23]$ and decreased hippocampal neurogenesis $[24,25]$. In the current study, we have shown that targeted disruption of ghrelin resulted in impaired memory performance of mice in the NOR test, which was rapidly reversed by ghrelin administration. Consistent with these findings, Diano et al. have reported that NOR performance was impaired in the GKO mice, which was rescued by peripheral ghrelin replacement via osmotic minipumps [8]. In addition, we also found that the GKO mice exhibited decreased spontaneous alternation behavior in the Y-maze, suggesting impairment in their spatial working memory. However, this functional impairment in the GKO mice was attenuated by ghrelin replacement therapy, indicating this peptide hormone exerts influences on brain structures responsible for learning and memory performance. It is unlikely that the learning impairment observed in the GKO mice could be attributed to decreased general locomotor activity, because there were no differences in total arm entries.

Several lines of evidence indicate that ghrelin plays an important role in learning and memory processes. Specifically, intracerebroventricular or intrahippocam- 
pal injection of ghrelin increases memory retention in rodents $[26,27]$. Ghrelin administered directly into the dorsal raphe nucleus (DRN) also increased memory retention via serotonergic inputs from the DRN [28]. Considering that adult hippocampal neurogenesis is correlated with spatial learning and memory process, decreased neurogenesis in GKO mice may contribute to impaired cognitive function. Indeed, memory impairment in ischemic injury is associated with neuronal cell loss in the hippocampus [29]. Several growth factors, including FGF-2, IGF-1 and VEGF, improve cognitive function through enhancement of generation or survival of new neurons in the hippocampus [3]. Therefore, it is likely that ghrelin-induced stimulation of neurogenesis in hippocampal progenitor cells may play an important role in improving cognitive functions. Another proposed mechanism through which ghrelin might enhance learning and memory is the promotion of hippocampal synaptic plasticity by augmenting dendritic spine formation and long term potentiation [8].

In summary, we have demonstrated that the absence of ghrelin results in reduced neurogenesis in the DG of adult hippocampus and impaired learning and memory, whereas systemic administration of ghrelin reverses these changes. These findings are significant because our data support the idea that ghrelin-induced hippocampal neurogenesis is required for the actions of ghrelin on hippocampus-related learning and memory processing. Therefore, ghrelin may be a plausible therapeutic approach for impaired cognitive function.

\section{Conflicts of Interest}

The authors declare that there is no conflict of interest that could be perceived as prejudicing the impartiality of the research reported.

\section{Acknowledgements}

This work was supported by the Korea Science and Engineering Foundation (KOSEF) grant funded by the Korea government (MEST) (No. 20120009383). We would like to extend our thanks to Dr. Rhonda D. Kineman (University of Illinois at Chicago, Chicago, IL) for proofreading and editing this manuscript.

\section{References}

1. Ming GL, Song H (2005) Adult neurogenesis in the mammalian central nervous system. Annu Rev Neurosci 28: 223-250.

2. Zhao C, Deng W, Gage FH (2008) Mechanisms and functional implications of adult neurogenesis. Cell 132: 645-660.

3. Grote HE, Hannan AJ (2007) Regulators of adult neurogenesis in the healthy and diseased brain. Clin Exp Pharmacol Physiol 34: 533-545.

4. Zhang W, Lin TR, Hu Y, Fan Y, Zhao L, et al. (2004) Ghrelin stimulates neurogenesis in the dorsal motor nucleus of the vagus. J Physiol 559: 729-737.

5. Zhang W, Hu Y, Lin TR, Fan Y, Mulholland MW (2005) Stimulation of neurogenesis in rat nucleus of the solitary tract by ghrelin. Peptides 26: 2280-2288.

6. Sato M, Nakahara K, Goto S, Kaiya H, Miyazato M, et al. (2006) Effects of ghrelin and des-acyl ghrelin on neurogenesis of the rat fetal spinal cord. Biochem Biophys Res Commun 350: 598-603.

7. Moon M, Kim S, Hwang L, Park S (2009) Ghrelin regulates hippocampal neurogenesis in adult mice. Endocr $J$ 56: 525-531.

8. Diano S, Farr SA, Benoit SC, McNay EC, da Silva I, et al. (2006) Ghrelin controls hippocampal spine synapse density and memory performance. Nat Neurosci 9: 381-
388.

9. Sato T, Kurokawa M, Nakashima Y, Ida T, Takahashi T, et al. (2008) Ghrelin deficiency does not influence feeding performance. Regul Pept 145: 7-11.

10. Broadbent NJ, Squire LR, Clark RE (2004) Spatial memory, recognition memory, and the hippocampus. Proc Natl Acad Sci U S A 101: 14515-14520.

11. Broadbent NJ, Gaskin S, Squire LR, Clark RE (2010) Object recognition memory and the rodent hippocampus. Learn Mem 17: 5-11.

12. Scholzen T, Gerdes J (2000) The Ki-67 protein: from the known and the unknown. J Cell Physiol 182: 311322.

13. Andrews ZB (2011) The extra-hypothalamic actions of ghrelin on neuronal function. Trends Neurosci 34: $31-40$.

14. Kojima M, Hosoda H, Date $Y$, Nakazato M, Matsuo H, et al. (1999) Ghrelin is a growth hormone-relesasing acylated peptide from stomach. Nature 402: 656-660.

15. Aberg MA, Aberg ND, Hedbacker H, Oscarsson J, Eriksson PS (2000) Peripheral infusion of IGF-I selectively induces neurogenesis in the adult rat hippocampus. J Neurosci 20: 2896-2903.

16. Aberg ND, Johansson I, Aberg MA, Lind J, Johansson UE, et al. (2009) Peripheral administration of GH 
induces cell proliferation in the brain of adult hypophysectomized rats. J Endocrinol 201: 141-150.

17. Lutter M, Sakata I, Osborne-Lawrence S, Rovinsky SA, Anderson JG, et al. (2008) The orexigenic hormone ghrelin defends against depressive symptoms of chronic stress. Nat Neurosci 11: 752-753.

18. Lee J, Seroogy KB, Mattson MP (2002) Dietary restriction enhances neurotrophin expression and neurogenesis in the hippocampus of adult mice. J Neurochem 80: 539-547.

19. Adams MM, Shi L, Linville MC, Forbes ME, Long AB, et al. (2008) Caloric restriction and age affect synaptic proteins in hippocampal CA3 and spatial learning ability. Exp Neurol 211: 141-149.

20. Fontan-Lozano A, Saez-Cassanelli JL, Inda MC, de los Santos-Arteaga M, Sierra-Dominguez SA, et al. (2007) Caloric restriction increases learning consolidation and facilitates synaptic plasticity through mechanisms dependent on NR2B subunits of the NMDA receptor. $J$ Neurosci 27: 10185-10195.

21. Gustafson D, Rothenberg E, Blennow K, Steen B, Skoog I (2003) An 18-year follow-up of overweight and risk of Alzheimer disease. Arch Intern Med 163: 1524-1528.

22. Rigamonti AE, Pincelli AI, Corra B, Viarengo R, Bonomo SM, et al. (2002) Plasma ghrelin concentrations in elderly subjects: comparison with anorexic and obese patients. $J$ Endocrinol 175: R1-R5.

23. Tschop M, Weyer C, Tataranni PA, Devanarayan V, Ravussin E, et al. (2001) Circulating ghrelin levels are decreased in human obesity. Diabetes 50: 707-709.

24. Kempermann G, GastD, GageFH(2002)Neuroplasticity in old age: sustained fivefold induction of hippocampal neurogenesis by long-term environmental enrichment. Ann Neurol 52: 135-143.

25. Lindqvist A, Mohapel P, Bouter B, Frielingsdorf H, Pizzo D, et al. (2006) High-fat diet impairs hippocampal neurogenesis in male rats. Eur J Neurol 13: 13851388.

26. Carlini VP, Ghersi M, Schioth HB, de Barioglio SR (2010) Ghrelin and memory: differential effects on acquisition and retrieval. Peptides 31: 1190-1193.

27. Carlini VP, Monzon ME, Varas MM, Cragnolini AB, Schioth HB, et al. (2002) Ghrelin increases anxietylike behavior and memory retention in rats. Biochem Biophys Res Commun 299: 739-743.

28. Carlini VP, Gaydou RC, Schioth HB, de Barioglio SR (2007) Selective serotonin reuptake inhibitor (fluoxetine) decreases the effects of ghrelin on memory retention and food intake. Regul Pept 140: 65-73.

29. Squire LR, Zola SM (1996) Ischemic brain damage and memory impairment: a commentary. Hippocampus 6: 546-552. 